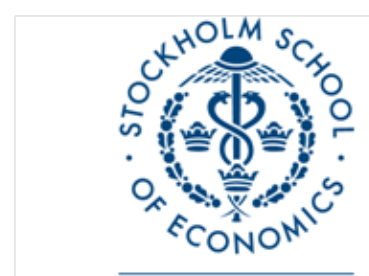

SITE STOCKHOLM INSTITUTE OF

TRANSITION ECONOMICS

SITE Working Paper No. 08, year 2010

\title{
Commitment in Alternating Offers Bargaining
}

\section{Topi Miettinen and Andrés Perea}

Stockholm Institute of Transition Economics

Stockholm School of Economics Sveavägen 65, P.O. Box 6501 SE - 11383 Stockholm 


\title{
Commitment in Alternating Offers Bargaining*
}

\author{
Topi Miettinen \\ Stockholm School of Economics \\ Andrés Perea \\ Maastricht University
}

This version: November 2009

\begin{abstract}
We extend the Ståhl-Rubinstein alternating-offer bargaining procedure to allow players, prior to each bargaining round, to simultaneously and visibly commit to some share of the pie. If commitment costs are small but increasing in the committed share, then the unique outcome consistent with common belief in future rationality (Perea, 2009), or more restrictively subgame perfect Nash equilibrium, exhibits a second mover advantage. In particular, as the smallest share of the pie approaches zero, the horizon approaches infinity, and commitment costs approach zero, the unique bargaining outcome corresponds to the reversed Rubinstein outcome $(\delta /(1+\delta), 1 /(1+\delta))$.

KEYWORDS: alternating offer bargaining, bargaining power, commitment, epistemic game theory, patience
\end{abstract}

JEL CODES: C73, C78, D84

Topi Miettinen: Stockholm School of Economics

P.O. Box 6501, Sveavägen 65, SE-113 83 Stockholm, Sweden.

topi.miettinen@hhs.se

Web: http://www2.hhs.se/SITE/homepages/topi.html

Andrés Perea: Maastricht University, Department of Quantitative Economics

P.O. Box 616, 6200 MD Maastricht, The Netherlands.

a.perea@maastrichtuniversity.nl

Web: http://www.personeel.unimaas.nl/a.perea/

${ }^{*}$ We would like to thank Tore Ellingsen and Jörgen Weibull for insightful comments. Financial support of Yrjö Jahnsson Foundation (Miettinen) gratefully acknowledged. 


\section{Introduction}

The main insight of Rubinstein's (1982) pioneering work on bargaining is that under complete information equilibrium strategies are determined by the relative impatience of the bargaining parties. In equilibrium there is an immediate efficient agreement. The proposer makes an offer so that the responder is indifferent between accepting the offer and rejecting it given the cost of waiting. Thus there is a first-mover advantage.

Subsequent research has pointed out two main sources of inefficiencies in complete information bargaining, non-stationarities (Fershtman and Seidmann, 1993; Compte and Jehiel, 2004; Li, 2007) and commitment strategies (Ellingsen and Miettinen (2008)). In the latter approach, negotiators end up with conflict when attempting to commit themselves to an aggressive bargaining stance and thereby forcing concessions from an uncommitted opponent. ${ }^{1}$ Ellingsen and Miettinen (2009) further illustrate how the chance of aggressively committing to stingy offers in a dynamic Rubinstein-like setting may lead to unavoidable conflict and/or asymmetries driven not only by patience, as predicted by Rubinstein, but also by the rate of decay of one's commitment power. $^{2}$

Like Miettinen and Ellingsen, we consider the effect of commitment strategies in a complete information alternating offer setting. We limit attention to the finite horizon alternating offer game (Ståhl, 1972) although we do study the infinite horizon limit. We model parties who can commit not to propose, or not to accept, any share smaller than specified in the commitment. Strategic commitment is assumed to incur small costs, increasing in the amount to which the party commits. The main difference to Ellingsen and Miettinen is that players do not commit directly to proposals, but rather commit to not offer, or commit to veto, any deal where their share is smaller than their commitment. In this sense, commitment resembles endogenous commitment analyzed in Fershtman and Seidmann (1993), Li (2007), and Miettinen (2009). ${ }^{3}$ Yet, rather than the smallest acceptable shares being determined by the bargaining history in some exogenously determined way, players can freely and simultaneously choose their commitments prior to each round of bargaining.

We show that the availability of such commitment strategies never leads to inefficiencies. As in Rubinstein (1982), deal is stroke in immediately. However, contrary to Rubinstein's outcome, there is now a second-mover advantage rather than a first-mover advantage! This is surprising, as both parties commit simultaneously at the beginning of every round, and there are no exogenous asymmetries in commitment technology. At the first round, the first-mover does best by refraining from committing altogether, whatever share the second-mover commits to. Knowing this, the second-mover will commit up to the share she would receive if (a) she were

\footnotetext{
${ }^{1}$ This point was initially discussed by Schelling (1956). Crawford (1982) showed that commitment strategies may lead to inefficienies when information is incomplete.

${ }^{2}$ See also Li (2009) who illustrates that efficient equilibria always exist if parties can update their commitments frequently enough.

${ }^{3}$ See also Comple and Jehiel (2004) on a model where endogenous outside options have a similar effect.
} 
to propose in the first round, and (b) there would be no possibility to commit in the first round. Thus, the presence of symmetric commitment strategies entirely reverses the bargaining power of the parties! In the limit, where the cost of commitment and the smallest indivisible share of the pie approach zero, and where the number of rounds approaches infinity, the outcome of the game approaches the reversed Rubinstein outcome, $(\delta /(1+\delta), 1 /(1+\delta))$.

We analyse the game using the concept of common belief in future rationality (Perea, 2009), meaning that both players always believe that the opponent will choose rationally now and in the future, that both players always believe that both players always believe that the opponent will choose rationally now and in the future, and so on. As Perea (2009) has shown, the strategies that may be chosen under common belief in future rationality can be computed by the algorithm of backwards dominance. Since every subgame perfect equilibrium of the game survives the backwards dominance procedure, it follows that the outcome described above is also the unique subgame perfect equilibrium outcome in the bargaining game.

Myerson (1991) and Abreu and Gul (2000) analyse a context where one party has incomplete information about the opponent's stubbornness not to accept anything less than an exogenously given share of the pie. The opponent can then use commitment tactics that exploit this incomplete information: However small the degree of uncertainty is, the incomplete information provides a bargainer with an option of strategically mimicking stubbornness in order to force concessions from the other party. This induces delay and conflict and a shift in the final sharing. Sequencing of moves and the implied bargaining power are not central to their analysis whereas in this paper these constitute a central focus. Moreover, outside options have been shown to eliminate the effect of stubbornness (Compte and Jehiel, 2002).

Outside options bear a close relation to the current complete information alternating offer bargaining model as well. It has been shown that, when a party, by opting out, gets a payoff that is inferior to the equilibrium payoff he would obtain in the game without outside options, then these latter have no effect on the equilibrium outcomes (Binmore et al. 1989). In our setting deliberately chosen commitment strategies influence bargaining outcomes exactly because they are chosen to force concessions superior to those in the Rubinstein outcome.

The result closest in spirit to ours is perhaps Dixit's (1980) extension of the Spence-Dixit excess capacity model. He shows that an incumbent firm, who nevertheless is presumed to play the role of the follower, can use the commitment, provided by an excess capacity investment, in seizing limited initiative back from the entrant.

The paper is organized as follows. In Section 2, we set up the model and the bargaining procedure. In Section 3 we present the concept of common belief in future rationality, and the associated algorithm of backwards dominance. In Section 4 we analyse the model with one round of bargaining. We will use it as a benchmark for our analysis of more than one round. Section 5 presents the general bargaining model with more than one round. Building upon our analysis of Section 4, we present the outcome that will result under common belief in future rationality. We also investigate the limit behavior of this outcome, when the commitment costs go to zero and the number of rounds goes to infinity. We conclude in Section 6 . 


\section{The Bargaining Procedure}

There are two players, 1 and 2, who must reach an agreement about the division of one unit of some good. We assume that the smallest amount is $1 / K$ for some integer number $K$. Let $X:=\{0,1 / K, 2 / K, \ldots, 1\}$. Hence, the set of possible divisions is given by

$$
D:=\left\{\left(x_{1}, x_{2}\right): x_{1}, x_{2} \in X \text { and } x_{1}+x_{2}=1\right\} .
$$

Players 1 and 2 use the following bargaining procedure, which can last for at most $N$ rounds.

Round 1: At the beginning, both players simultaneously choose commitment levels $c_{1}, c_{2} \in$ $X$. The commitment levels become known to both players, and player 1 proposes a division $\left(x_{1}, x_{2}\right) \in D$ with $x_{1} \geq c_{1}$. Subsequently, player 2 decides whether to accept or reject the proposal under the condition that he can only accept offers with $x_{2} \geq c_{2}$. If he accepts, $\left(x_{1}, x_{2}\right)$ is the final outcome. If he rejects, the game moves to round 2 .

Round 2: At the beginning, both players simultaneously choose new commitment levels $c_{1}, c_{2} \in$ $X$. Afterwards, player 2 proposes a division $\left(x_{1}, x_{2}\right) \in D$ with $x_{2} \geq c_{2}$. Subsequently, player 1 decides whether to accept or reject $\left(x_{1}, x_{2}\right)$, under the condition that he can only accept offers with $x_{1} \geq c_{1}$. If he accepts, $\left(x_{1}, x_{2}\right)$ is the final outcome. If he rejects, the game moves to round 3 .

Round 3: This is a repetition of round 1. And so on.

This bargaining procedure goes on until an agreement is reached, or the process enters round $N+1$. In round $N+1$, a given division $\left(y_{1}, y_{2}\right) \in D$ with $y_{1}, y_{2}>0$ is realized.

We assume that both players incur a cost for commitment. More precisely, if player $i$ commits to an amount $c_{i}$, this will cost him $\lambda c_{i}$, where $\lambda$ is some small positive number. For convenience, we assume that $\lambda$ is the same for both players. We finally assume that both players discount future payoffs by a common discount factor $\delta$.

So, in view of all the above, the players' utilities are as follows: If the players reach an agreement on division $\left(x_{1}, x_{2}\right)$ in round $n$, then the utility for player $i$ is

$$
\delta^{n-1} x_{i}-\lambda\left(c_{i}^{1}+\delta c_{i}^{2}+\ldots+\delta^{n-1} c_{i}^{n}\right),
$$

where $c_{i}^{k}$ is the commitment level chosen at round $k$. If the game reaches round $N+1$, his utility would be

$$
\delta^{N} y_{i}-\lambda\left(c_{i}^{1}+\delta c_{i}^{2}+\ldots+\delta^{N-1} c_{i}^{N}\right) .
$$

In order to avoid uninteresting indifferences, we assume that $\delta$ is such that a player is never indifferent between two outcomes that are realized at two different rounds. Note that for every open interval $(a, b)$ in $[0,1]$, we can always find such a $\delta$ that lies in $(a, b)$, since there are only finitely many rounds, and finitely many divisions and commitment levels at every round. By choosing $\delta$ is this way, we guarantee that a player will never be indifferent between accepting 
and rejecting an offer. This, eventually, will lead to a unique outcome in the bargaining game, which makes our analysis more transparent.

Within our bargaining procedure above, the interpretation of the commitment levels is thus that the proposer commits to never offer less than his commitment level for himself, whereas the responder commits to reject any offer that would give him less than his commitment level.

\section{Common Belief in Future Rationality}

The concept we use to analyse the game is common belief in future rationality (Perea (2009)). That is, we assume that a player always believes that his opponent will choose rationally now and in the future, that a player always believes that his opponent always believes that he will choose rationally now and in the future, and so on. For a formal definition of this concept within an epistemic model the reader is referred to Perea (2009). For finite dynamic games with almost perfect information, Perea (2009) has shown that the concept can be characterized by an elimination procedure called backwards dominance. More precisely, for such games the procedure selects exactly those strategies that can rationally be chosen under common belief in future rationality.

Here, by a dynamic game with almost perfect information we mean a game where at every stage one or more players make a choice simultaneously, and where these choices become known to everyone before the next stage starts. It is easily seen that our bargaining procedure is a finite dynamic game with almost perfect information. Hence, we can use backwards dominance to select those strategies that can rationally be chosen under common belief in future rationality.

The backwards dominance procedure works as follows: We start at the ultimate subgames, that is, those subgames after which the game is over. At each of those subgames, we restrict to strategies that reach this subgame, and apply iterated strict dominance (or, iterated elimination of strictly dominated strategies) to this restricted game.

We then move to penultimate subgames, that is, subgames after which either the game is over or an ultimate subgame starts. At each of those subgames, we restrict to strategies that reach this subgame and that have not been eliminated yet by the procedure. We then apply iterated strict dominance to these restricted games.

And so on, until we reach the beginning of the game. There, we restrict to strategies that have not been eliminated yet, and apply iterated strict dominance to this restricted game. The strategies that survive the final round of iterated strict dominance at the beginning of the game are said to survive the backwards dominance procedure.

For finite dynamic games with almost perfect information, subgame perfect equilibrium is a strict refinement of common belief in future rationality. That is, every strategy that is optimal in a subgame perfect equilibrium can also be chosen rationally under common belief in future 
rationality, but not vice versa (see Perea (2009)). If we apply common belief in future rationality to games with perfect information, we obtain the usual backwards induction procedure.

\section{The Case of One Round}

We start with the easiest case, namely when there is only one round of bargaining. For this case, we already encounter a surprising result: Under common belief in future rationality (and hence also under subgame perfect equilibrium) the proposer faces a first-mover disadvantage, rather than a first-mover advantage. Actually, we can say a little more, namely the proposer gets exactly what he would obtain as a responder in the procedure without commitment. So, introducing the possibility to commit reverses the outcome completely! All this is obtained under the assumption that the commitment costs are sufficiently small. More precisely, we require $\lambda<1-\delta$.

Theorem 4.1. (Case of one round) Consider the procedure with only one round of bargaining, and suppose that $\lambda<1-\delta$. Then, under common belief in future rationality, player 1 chooses commitment level 0 , player 2 chooses commitment level $1-\overline{\delta y_{1}}$, player 1 proposes $\left(\overline{\delta y_{1}}, 1-\overline{\delta y_{1}}\right)$ and player 2 accepts.

Here, $\bar{x}$ denotes the smallest number in $X$ larger than, or equal to, $x$. Remember that $\left(y_{1}, y_{2}\right)$ is the outcome if the proposal is rejected. So, player 1 , the proposer, gets the minimal amount he would still accept, whereas player 2 , the responder, gets all the surplus! Notice that in the classical bargaining procedure without commitment, this would be exactly the outcome when player 2 would be the proposer and player 1 the responder.

Proof. For every pair $\left(c_{1}, c_{2}\right)$ of commitment levels, the subgame that starts after $\left(c_{1}, c_{2}\right)$ is a game with perfect information. Hence, applying backwards dominance to this subgame is the same as using backwards induction. After every $\left(c_{1}, c_{2}\right)$, the backwards induction outcome is as follows:

1. If $c_{1}+c_{2}>1$, or $c_{1}>1-\delta y_{2}$, then player 2 will reject any proposal by player 1 . Hence, the outcome will be $\left(y_{1}, y_{2}\right)$, with utility $\delta y_{1}-\lambda c_{1}$ for player 1 , and utility $\delta y_{2}-\lambda c_{2}$ for player 2 .

2. If $c_{2}>1-\delta y_{1}$, then player 1 does not want to make any offer that player 2 would accept. Hence, the outcome will be $\left(y_{1}, y_{2}\right)$, with utility $\delta y_{1}-\lambda c_{1}$ for player 1 , and utility $\delta y_{2}-\lambda c_{2}$ for player 2 .

3. Suppose that $c_{1}+c_{2} \leq 1$ and $\delta y_{2}<c_{2}<1-\delta y_{1}$. Then, the best that player 1 can do is to offer player 2 precisely $c_{2}$, which player 2 would accept. So, the outcome would be $\left(1-c_{2}, c_{2}\right)$, with utility $1-c_{2}-\lambda c_{1}$ for player 1 , and utility $c_{2}-\lambda c_{2}$ for player 2 . 


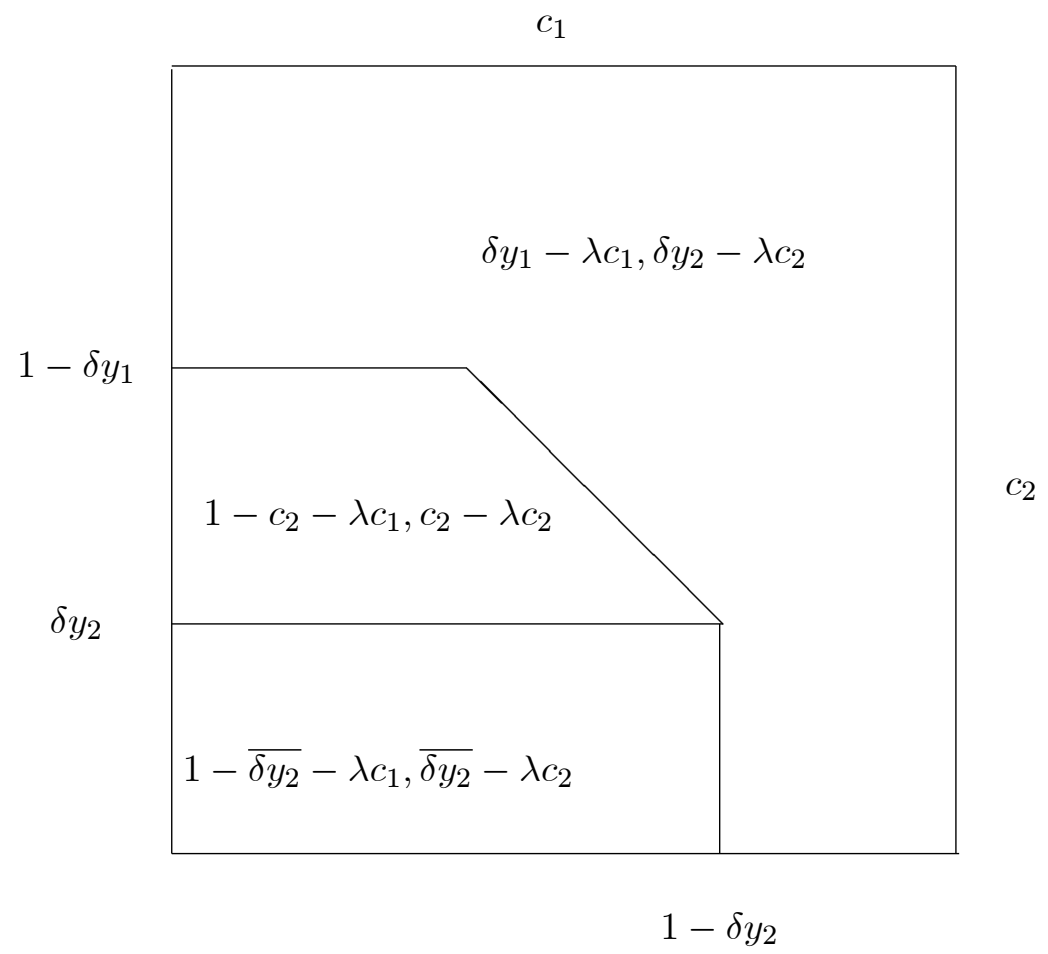

Figure 1: The case of one round: Backwards induction utilities after every pair $\left(c_{1}, c_{1}\right)$

4. Suppose that $c_{1}<1-\delta y_{2}$ and $c_{2}<\delta y_{2}$. Then, the best that player 1 can do is to offer player 2 exactly $\overline{\delta y_{2}}$, which player 2 would accept. So, the outcome would be $\left(1-\overline{\delta y_{2}}\right.$, $\left.\overline{\delta y_{2}}\right)$, with utility $1-\overline{\delta y_{2}}-\lambda c_{1}$ for player 1 , and utility $\overline{\delta y_{2}}-\lambda c_{2}$ for player 2 .

It can easily be seen that this covers all possible cases. In Figure 1 we have depicted the backwards induction utilities for both players after every possible pair $\left(c_{1}, c_{2}\right)$. So, Figure 1 represents exactly the restricted game that the backwards dominance procedure would consider at the beginning of the game. In order to finish the backwards dominance procedure, we must apply iterated strict dominance to the game in Figure 1.

From player 1's utilities in Figure 1 it is easily verified that, for every $c_{2}$, player 1's utility is decreasing in his commitment level $c_{1}$. This means, however, that $c_{1}=0$ strictly dominates every other $c_{1}$ for player 1 . So, we eliminate all $c_{1}>0$ for player 1 , which leaves only $c_{1}=0$. But then, in the reduced game that remains, player 2's best choice is $c_{2}=1-\overline{\delta y_{1}}$. Here, we use the assumption that $\lambda<1-\delta$. As we have seen above, the best that player 1 can do in this 
case is to propose $\left(\overline{\delta y_{1}}, 1-\overline{\delta y_{1}}\right)$, which player 2 would accept. So, by applying the backwards dominance procedure, we obtain that player 1 chooses commitment level $c_{1}=0$, player 2 chooses $c_{2}=1-\overline{\delta y_{1}}$, player 1 proposes $\left(\overline{\delta y_{1}}, 1-\overline{\delta y_{1}}\right)$ and player 2 accepts. This completes the proof.

\section{The Case of More Rounds}

We now turn to the case of more than one round. Also in this case, common belief in future rationality leads to a unique outcome, where the proposer at round 1 faces a first-mover disadvantage, rather than a first-mover advantage. Actually, when the commitment cost $\lambda$ tends to zero, then the first proposer gets exactly what he would obtain as the first responder in the procedure without commitment, and vice versa. So, again, introducing the possibility to commit completely reverses the outcome as $\lambda$ tends to zero! As every subgame perfect equilibrium satisfies common belief in future rationality (see Perea (2009)), it follows that this outcome is also the unique subgame perfect equilibrium outcome in the game.

Theorem 5.1. (Case of more than one round) Suppose that the bargaining procedure consists of $N$ potential rounds, and that $\lambda<1-\delta$. Let $p$ denote the proposer at round 1 , and $r$ the responder at round 1 . Then, common belief in future rationality leads to a unique outcome, namely at round 1 proposer $p$ commits to $c_{p}=0$, responder $r$ commits to $c_{r}=x_{r}^{N}$, proposer $p$ proposes the division $\left(x_{p}^{N}, x_{r}^{N}\right)$ and responder $r$ accepts, where $x_{p}^{N}, x_{r}^{N}$ are recursively given by

$$
\begin{aligned}
x_{p}^{1} & =\overline{\delta y_{p}}, x_{r}^{1}=1-\overline{\delta y_{p}}, \\
x_{p}^{N} & =\overline{\delta(1-\lambda) x_{r}^{N-1}}, \text { and } x_{r}^{N}=1-\overline{\delta(1-\lambda) x_{r}^{N-1}},
\end{aligned}
$$

for every $N \geq 2$.

If we let $\lambda$ tend to zero, then the recursive equations above would exactly yield the outcomes for the players in the procedure without commitment, but with the roles of the proposer and responder reversed! If the size of the smallest slice $1 / K$ is small, then the amounts $x_{p}^{N}$ and $x_{r}^{N}$ are approximately equal to

$$
x_{p}^{N} \approx \frac{\delta(1-\lambda)+(-1)^{n} \delta^{n}(1-\lambda)^{n}}{1+\delta(1-\lambda)}+(-1)^{n-1} \delta^{n}(1-\lambda)^{n-1} y_{p}
$$

and

$$
x_{r}^{N} \approx \frac{1-(-1)^{n} \delta^{n}(1-\lambda)^{n}}{1+\delta(1-\lambda)}-(-1)^{n-1} \delta^{n}(1-\lambda)^{n-1} y_{p} .
$$

Recall that $y_{p}$ is the amount that player $p$ would get at the end of the game, when all proposals have been rejected. These approximations are obtained by setting $\bar{x}=x$ in the recursive equations above, and solving them. If the number of rounds $N$ becomes very large, then

$$
x_{p}^{N} \approx \frac{\delta(1-\lambda)}{1+\delta(1-\lambda)} \text { and } x_{r}^{N} \approx \frac{1}{1+\delta(1-\lambda)}
$$


which shows that there is a clear first-mover disadvantage. If in addition the commitment cost $\lambda$ would tend to zero, then in the limit we would obtain the reversed Rubinstein outcome

$$
x_{p}^{N} \approx \frac{\delta}{1+\delta} \text { and } x_{r}^{N} \approx \frac{1}{1+\delta} .
$$

Proof of Theorem 5.1. We prove the statement by induction on the number of rounds. If $N=1$, then the statement follows immediately from Theorem 4.1.

Now, assume that $N \geq 2$, and that the statement holds for the procedure with $N-1$ rounds. Let $p$ be the proposer at round 1 , and $r$ the responder at round 1. Suppose that the proposal at round 1 would be rejected. Then, the subgame that starts at round 2 is a procedure with $N-1$ rounds, where $r$ is the first proposer and $p$ is the first responder. The commitment costs incurred at round 1 are sunk costs, and therefore do not affect the analysis in this subgame. By our induction assumption we know that in this subgame, common belief in future rationality (or, equivalently, the backwards dominance procedure) leads to a unique outcome: player $r$ chooses commitment level $c_{r}=0$, player $p$ chooses commitment level $c_{p}=x_{r}^{N-1}$, player $r$ proposes $x_{p}^{N-1}$ for himself and $x_{r}^{N-1}$ for player $p$, and player $p$ accepts. The corresponding utilities would be $x_{r}^{N-1}-\lambda x_{r}^{N-1}=(1-\lambda) x_{r}^{N-1}$ for player $p$ and $x_{p}^{N-1}$ for player $r$.

Let us now move to round 1 , the beginning of the game. If we apply the backwards dominance procedure, then we restrict to strategies that have not been eliminated yet, and perform iterated strict dominance within this restricted game. By our induction assumption, the strategies that have not been eliminated yet are such that, whenever the proposal at round 1 is rejected, then the discounted utility for $p$ is $\delta(1-\lambda) x_{r}^{N-1}$, and the discounted utility for $r$ is $\delta x_{p}^{N-1}$. By a similar argument as in the proof of Theorem 4.1, we can then conclude that the restricted game at round 1 is given by Figure 2. The only change compared to the proof in Theorem 4.1 is that we substitute $(1-\lambda) x_{r}^{N-1}$ for $y_{1}$, and substitute $x_{p}^{N-1}$ for $y_{2}$. In Figure 2 , the first utility always corresponds to player $p$, and the second utility to player $r$.

From Figure 2, it can easily be concluded that proposer $p$ 's utility is strictly decreasing in his commitment level $c_{p}$. Hence, all choices but $c_{p}=0$ are strictly dominated for player $p$. So, we obtain a reduced game in which player $p$ only chooses $c_{p}=0$. But then, using the assumption that $\lambda<1-\delta$, we see that player $r$ 's best choice is $c_{r}=1-\overline{\delta(1-\lambda) x_{r}^{N-1}}$. So, the backwards dominance procedure (and hence also common belief in future rationality) leads to a unique outcome, in which at round 1 player $p$ commits to $c_{p}=0$, player $r$ commits to $c_{r}=1-\overline{\delta(1-\lambda) x_{r}^{N-1}}$, player $p$ proposes $\overline{\delta(1-\lambda) x_{r}^{N-1}}$ for himself, player $p$ proposes $1-\overline{\delta(1-\lambda) x_{r}^{N-1}}$ for player $r$, and player $r$ accepts. Since $x_{p}^{N}=\overline{\delta(1-\lambda) x_{r}^{N-1}}$ and $x_{r}^{N}=1-\overline{\delta(1-\lambda) x_{r}^{N-1}}$, the statement of the theorem follows for $N$ rounds. By induction on $N$, the statement holds for every $N$, and hence the proof is complete. 


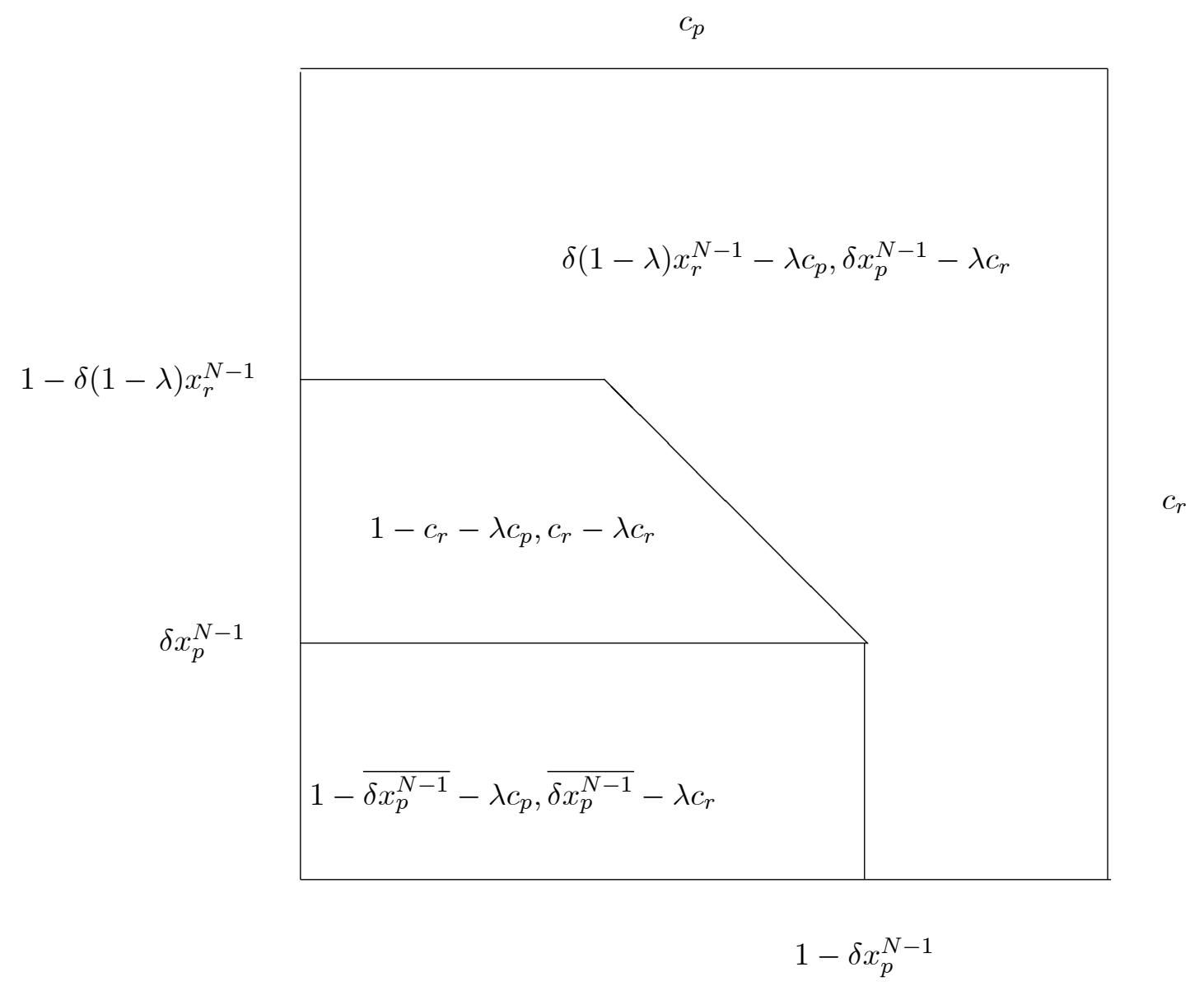

Figure 2: The restricted game at round 1 


\section{Concluding Remarks}

\subsection{Commitment Costs}

In our model we have assumed that the commitment costs for both players are given by $\lambda c$, where $c$ is the amount committed to, and $\lambda$ is some fixed number less than $1-\delta$. In fact, we do not really need this specific functional form for the commitment costs. Instead, we could assume that the commitment costs are given by a more general function $\gamma(c)$, where $\gamma(0)=0$, the function $\gamma$ is strictly increasing in the commitment level $c$, and $\gamma(1) \leq 1-\delta$. The reader may verify that under these assumptions, common belief in future rationality would again lead to a unique outcome, in which the proposer at round 1 faces a first-mover disadvantage. The outcome can be computed by a recursive formula similar to the one used in Theorem 5.1. Also under these assumptions we would obtain the reversed Rubinstein outcome $(\delta /(1+\delta), 1 /(1+\delta))$ if we let the number of rounds go to infinity, let the size of the smallest slice go to zero, and let the commitment costs go to zero. However, in the paper we have chosen the specific functional form $\lambda c$ for the commitment costs as to keep the presentation and the analysis as simple as possible.

\subsection{Common Belief in Future Rationality}

The reader may wonder why we have not chosen the more traditional concept of subgame perfect equilibrium, instead of common belief in future rationality, to analyse the game. There are two reasons.

First, common belief in future rationality is a more basic concept than subgame perfect equilibrium, as it does not impose any equilibrium condition. It only requires that a player always believes that his opponent will choose rationally now and in the future, that he always believes that his opponent always believes that he will choose rationally now and in the future, and so on. The concept of subgame perfect equilibrium also imposes these conditions, but in addition requires some equilibrium conditions that are harder to justify, and which are not assumed by common belief in future rationality.

Second, using common belief in future rationality as a concept makes our Theorem 5.1 stronger. Namely, common belief in future rationality is a weaker concept than subgame perfect equilibrium. In fact, every subgame perfect equilibrium satisfies common belief in future rationality, but not vice versa. Therefore, our Theorem 5.1 implies that the outcome described there is also the unique subgame perfect equilibrium outcome. However, the statement is stronger than this: We do not need the equilibrium condition to arrive at this outcome. Imposing only common belief in future rationality is already enough.

\section{References}

[1] Abreu, D., F. Gul (2000): "Bargaining and Reputation" Econometrica, 68, 85-118. 
[2] Binmore, K., Shaked, A., Sutton, J. (1989): "An Outside Option Experiment" Quarterly Journal of Economics, 104, 753-770.

[3] Compte, O., P. Jehiel (2002): "On the Role of Outside Options in Bargaining with Obstinate Parties", Econometrica 70, 1477-1517

[4] Compte, O., P. Jehiel (2004): "Gradualism in Bargaining and Contribution Games", Review of Economic Studies 71, 975-1000.

[5] Crawford, V.P. (1982): "A Theory of Disagreement in Bargaining" Econometrica, 50, 607637.

[6] Dixit, A. (1980): "The Role of Investment in Entry-Deterrence", Economic Journal, 90, 95-106.

[7] Ellingsen, T., T. Miettinen (2008): "Commitment and Conflict in Bilateral Bargaining", American Economic Review 98, 1629-1635.

[8] Ellingsen, T., T. Miettinen (2009): "Temporary Commitment in Dynamic Bargaining" Stockholm School of Economics, mimeo.

[9] Fershtman, C., D. Seidmann (1993): "Deadline Effects and Inefficient Delay in Bargaining with Endogenous Commitment." Journal of Economic Theory 60, 306-321.

[10] Li, D. (2007): "Bargaining with History-Dependent Preferences", Journal of Economic Theory 136, 695-708.

[11] Li, D. (2009): :"Commitment and Compromise in Dynamic Bargaining", Chinese University of Hong Kong, mimeo.

[12] Miettinen, T. (2009): "History-dependent Reciprocity in Alternating-offer Bargaining" Stockholm School of Economics, mimeo.

[13] Myerson, R. (1991). Game Theory: Analysis of Conflict, Harvard University Press, Cambridge, MA/USA.

[14] Perea, A. (2009): "Belief in the Opponents' Future Rationality" Working paper, Available at: http://www.personeel.unimaas.nl/a.perea/

[15] Rubinstein, A. (1982): "Perfect Equilibrium in a Bargaining Model" Econometrica 50, 97110.

[16] Schelling, T. (1956): "An Essay on Bargaining." American Economic Review 46, 281-306.

[17] Ståhl, I. (1972). Bargaining Theory. Stockholm School of Economics, Stockholm, Sweden. 